$1986-12$

\title{
Multidimensional Scaling in Counseling Research and Practice
}

Mark L. Davison

P. Scott Richards

Brigham Young University - Provo, scott_richards@byu.edu

James B. Rounds Jr.

Follow this and additional works at: https://scholarsarchive.byu.edu/facpub

Part of the Student Counseling and Personnel Services Commons

\section{Original Publication Citation}

Davison, M.L., Richards, P.S., \& Rounds, J.B. (1986). Multidimensional scaling in counseling research and practice. Journal of Counseling and Development, 65, 178-184.

\section{BYU ScholarsArchive Citation}

Davison, Mark L.; Richards, P. Scott; and Rounds, James B. Jr., "Multidimensional Scaling in Counseling Research and Practice" (1986). Faculty Publications. 3872.

https://scholarsarchive.byu.edu/facpub/3872 


\section{Multidimensional Scaling in}

\section{Counseling Research}

and Practice

MARK L. DAVISON, P. SCOTT RICHARDS, and JAMES B. ROUNDS, JR.

The authors briefly describe the use of multidimensional scaling (MDS) in counseling. They discuss types of data that have been analyzed using $M D S$, kinds of MDS analyses, and the interpretation of MDS results and review MDS research relevant to vocational, family, group, and individual counseling. They also briefly discuss potential applications in counseling practice.

M ultidimensional scaling (MDS) is a statistical technique that can be used in mapping the structure of objects as they are perceived by clients and counselors. It has become increasingly popular in recent years, and there are now several full-length textbooks available (Coxon, 1982; Davison, 1983; Kruskal \& Wish, 1978; Schiffman, Reynolds, \& Young, 1981). Counseling has benefited from the use of MDS for studying issues such as dimensions of vocational interest, the perceived structure of occupations, interpersonal perceptions and their progressive changes in a therapy group, and the dimensions of psychopathology. As yet, counseling practice has benefited little from direct application of MDS. In our opinion, however, MDS holds genuine promise as a clinical tool for preinterview assessment, for tracking intervention outcomes, and for client feedback.

In this article we describe a multidimensional scaling analysis, discussing the nature of input data, the assumptions underlying the analysis, and the output. We briefly compare MDS to its two major alternatives, factor and cluster analysis, and review existing applications of MDS in counseling research. Because space considerations force this review to be highly selective, we have prepared an extensive bibliography of counseling articles that can be obtained from the senior author. Jones (1983), Rounds and Zevon (1983), and Shoben (1983) also included extensive reviews of MDS research in social, vocational, and cognitive psychology, respectively. We also briefly discuss the development of MDS approaches to pre-interview assessments, the tracking of intervention outcomes, and client feedback.

\section{MULTIDIMENSIONAL SCALING ANALYSES}

Four steps are required in designing any MDS study: sampling participants and stimuli, choosing an appropriate data collection technique, selecting one of several MDS analyses, and interpreting the output. We first survey the alternatives at each of these four choice points and then illustrate these issues with an example from vocational counseling and research.

\section{Participants and Stimuli}

The first step in an MDS analysis is to decide what participants and what stimuli are to be studied. This step creates a double sampling problem. If one wants to generalize to a population beyond the particular participants studied, it is important to draw those participants randomly. The same consideration ap- plies to stimuli. That is, if one wants to generalize beyond the sample of stimuli studied, then one must draw the stimuli randomly or at least representatively. The stimuli actually sampled will almost certainly have an influence on the dimensions that emerge in the MDS structure. An attribute is unlikely to appear in the solution if the stimuli do not vary on that attribute. For instance, if one includes only professional occupations in a study of high school students' perceptions of occupations, then prestige is unlikely to emerge as a dimension. On the other hand, if the sample includes occupations that vary in prestige (e.g., medical doctor, rehabilitation counselor, retail sales clerk, janitor), then a prestige dimension may well emerge.

\section{Input Data}

The second step is to choose the kind of data that will be collected from the participants. The basic data for an MDS analysis consist of one or more proximity matrices, such as that in Table 1. Each element of a proximity matrix represents the measured similarity (or dissimilarity) between a pair of objects. In Table 1 the numbers represent hypothetical average ratings of similarity on a 5-point rating scale ranging from highly dissimilar (1) to highly similar (5). For instance, the element in row 3 , column 1 indicates that the occupational pair (pharmacist, librarian) received an average rating of 1.3 out of a possible 5.0 , suggesting that participants did not see much resemblance between these two jobs.

The rather broad definition of proximity datum - a measure for a pair of objects indicating the degree of similarity or dissimilarity between them-can be operationalized in many ways. Not surprisingly, users of MDS have employed a wide variety of experimental methods to gather their data and to measure the similarity (or dissimilarity) of their stimulus objects. These data collection techniques can be classified into three groups: direct similarity judgments, probability measures, and profile similarity measures.

In direct similarity judgment techniques, the researcher presents participants with a pair of objects and asks them to rate

TABLE 1

\section{Hypothetical Similarity Data for Five Occupations}

\begin{tabular}{lccccc}
\hline \hline & Librarian & Teacher & Pharmacist & Architect & Electrician \\
\hline Librarian & - & 4.6 & 1.3 & 2.3 & 1.8 \\
$\begin{array}{l}\text { Teacher } \\
\text { Pharma- }\end{array}$ & 4.6 & - & 1.4 & 2.0 & 2.1 \\
$\quad$ cist & 1.3 & 1.4 & - & 1.1 & 3.1 \\
Architect & 2.3 & 2.0 & 1.1 & - & 1.3 \\
Electrician & 1.8 & 2.1 & 3.1 & 1.3 & - \\
\hline
\end{tabular}


(or express in some other form) the similarity or dissimilarity of those objects. A typical rating could appear as follows: Librarian, Pharmacist
Highly Dissimilar 2
Highly Similar

4 5
Such ratings do not specify the attributes on which participants are to rate the pair of occupations, and hence the task leaves the participant free to judge the pair on whatever features he or she chooses. These direct similarity judgments are usually obtained for all possible stimulus pairs (e.g., all possible pairs of occupations), and the ratings then become the proximity data.

With some stimulus sets, it may be more appropriate to rate on a concept other than similarity per se. If the stimuli are family members, for instance, clients could rate pairs of family members on the closeness of their relationship rather than on similarity. Properly phrasing the rating concept is very important in obtaining judgments of direct proximity.

Measures based on probability usually represent the probability that the two stimuli will occur together. For example, in a study examining the structure of the American Association for Counseling and Development's divisions, a measure of similarity could be obtained for each pair of divisions by counting the number of people who belonged to both divisions and converting these frequencies into proportions. Presumably, the more similar two divisions are, the more members they have in common. Rosenberg (1982) derived a proximity matrix for trait adjectives by instructing participants to sort traits into several categories in which each category corresponds to one of the participant's friends. A similarity measure can be obtained for pairs of traits by counting the number of friends who are assigned both traits and converting this frequency count into a proportion. (See Rosenberg, 1982, for a more complex proximity measure that can be derived using the same task.)

Finally, there are profile similarity measures, such as the correlation coefficient. Correlations have been used frequently in studies of tests and test items. In a typical study the objects are personality or ability tests and the correlation between two tests is their measure of similarity.

\section{Analyses}

Although MDS statistical analyses differ in many respects, the two most important distinctions for our purposes are the metric versus nonmetric and the two- versus three-way distinction. Metric analyses assume at least interval level properties for the data. Nonmetric analyses assume ordinal data. Because they can be applied to ordinal data, nonmetric analyses are applicable to a wider array of data than are metric analyses. There are several good computer programs that can perform nonmetric analyses; the best known are ALSCAL (Young \& Lewyckyj, 1979), KYST (Kruskal, Young, \& Seery, 1973), and MINISSA (Lingoes, 1973).

The second distinction concerns two- versus three-way analyses. In a two-way analysis, the input consists of just one proximity matrix, such as a correlation matrix. In a three-way (or individual differences) analysis, the input consists of more than one proximity matrix, with each matrix representing a participant. Unlike a two-way analysis, a three-way analysis provides information about how various participants seem to use stimulus dimensions differently in arriving at their judgments. Programs for analyzing two- or three-way data include ALSCAL (Young \& Lewyckyj, 1979), INDSCAL (Carroll \& Chang, 1970), MULTISCALE (Ramsey, 1978), and SINDSCAL (Pruzansky, 1975); all are metric except ALSCAL.

\section{Output}

The output takes different forms in the many different MDS programs. All programs, however, yield estimates of stimulus coordinates along dimensions. The three-way (or individual differences) analysis also produces a table of dimension weights for participants. In addition, MDS analysis produces a fit measure. The following is an explanation of how to interpret the stimulus coordinates, participant weights, and fit measure.

Stimulus coordinates. The major portion of the output consists of the stimulus coordinates, which are presented as a table (e.g., Table 2) and as a series of plots (e.g., Figure 1). (Another graph would be needed to represent visually the coordinates for $\mathrm{Di}$ mensions 3 and 4.) Figure 1 is simply a graphical representation of the Dimension 1 and Dimension 2 stimulus coordinates in Table 2. For example, the (aircraft) mechanic stimulus, which has coordinates of .17 along Dimension 1 and .16 along Dimension 2 in Table 2, has been plotted in Figure 1 at a location that lies .17 units to the right of the origin along the horizontal axis

TABLE 2

Four-dimensional INDSCAL Solution for All Participants

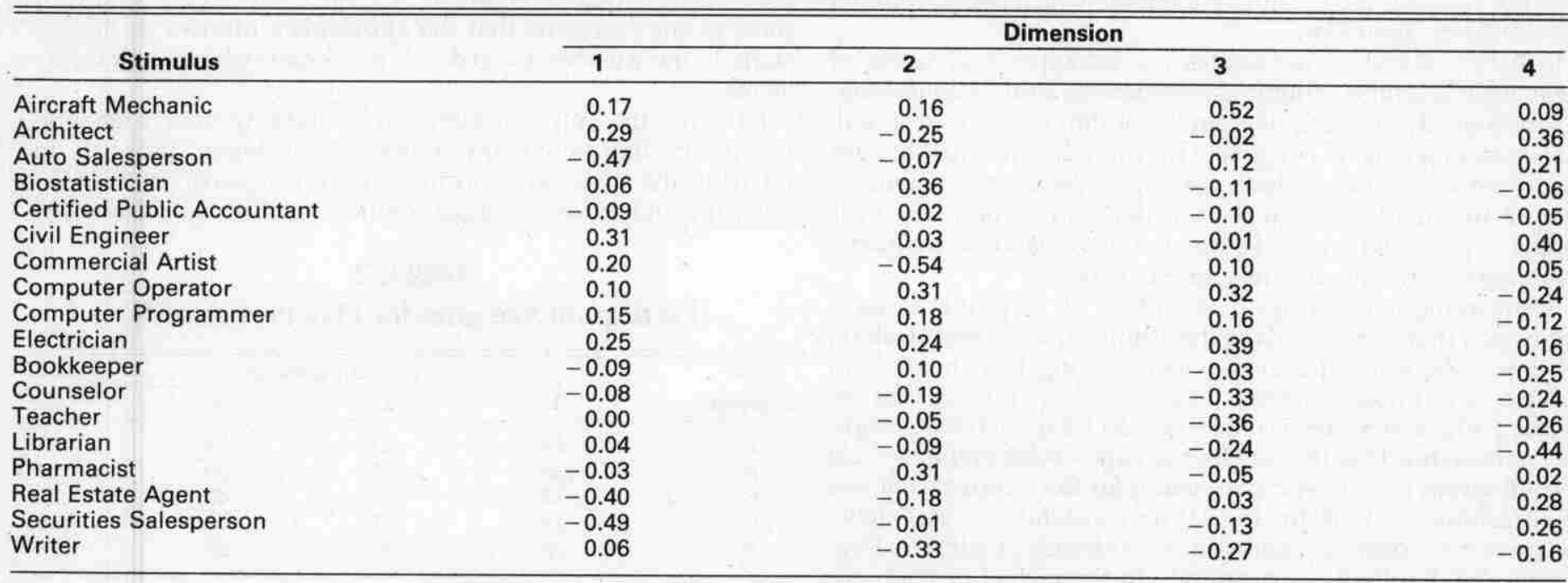




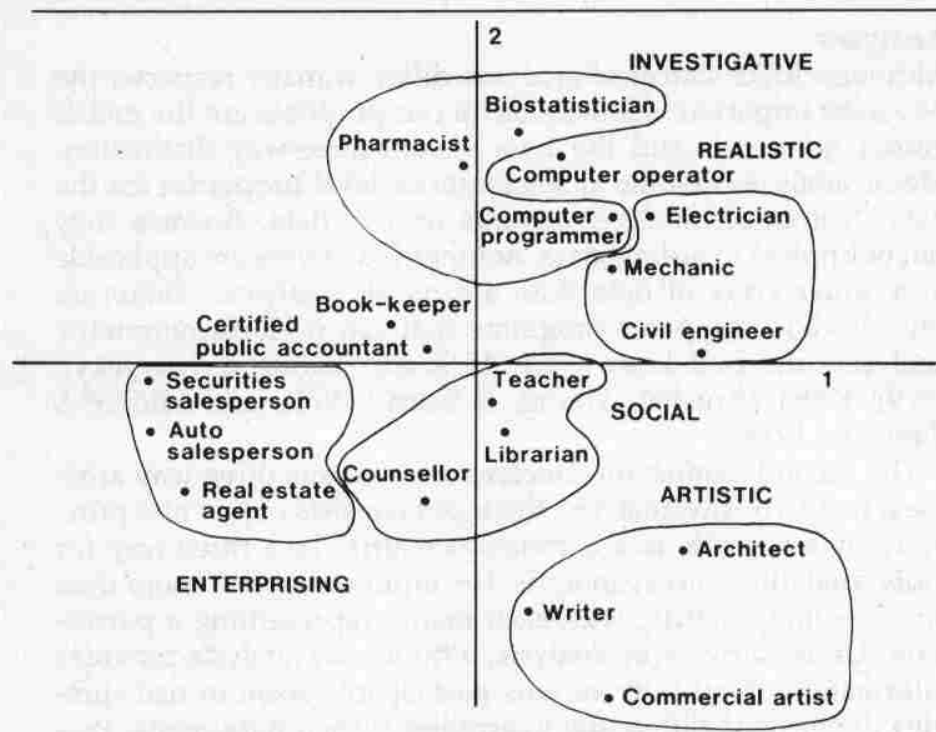

FIGURE 1

\section{Dimension 1, the Production-Sales Dimension Plotted Against Dimension 2, the Scientific-Artistic Dimension}

(Dimension 1) and .16 units above the origin along the vertical axis (Dimension 2). To understand the interpretation of stimulus coordinates, one must understand the analogy on which MDS is built.

Multidimensional scaling employs an elaborate analogy between the psychological concept of dissimilarity and the geometric concept of distance. Dissimilar stimuli are represented as geometrically distant. In Figure 1, for instance, Teacher and Librarian are located near each other because they were rated by participants as quite similar. On the other hand, Biostatistician and Real Estate Agent occupy distant locations at opposite corners of the plot because participants rated them as being highly dissimilar. In ordinary speech, people implicitly recognize the dissimilarity-distance analogy when they speak of two things that resemble each other highly as being "close" or when they speak of two things that are quite unlike each other as being "not at all close." In an MDS analysis, each stimulus is assigned coordinates along dimensions in such a way that stimuli that are similar, according to the proximity data, are located near each other and stimuli that are dissimilar are located far apart. By visually scanning the MDS plot(s) and examining the distance between the stimuli, one can tell which pairs of stimuli are similar or dissimilar.

In MDS, stimulus coordinates are interpreted in terms of meaningful stimulus groupings or orderings along a dimension. A substantively meaningful grouping of stimuli is a set of stimuli that cluster together in a region of the multidimensional solution space and hence are similar according to the data. In addition, they share some common feature that can account for their similar representation. In Figure 1, the Holland occupational groupings represent such meaningful clusters.

A meaningful ordering of stimuli is an ordering along a dimension that corresponds to the arrangement of stimuli along an important attribute. For instance, along Dimension 4 in Table 2, the highest paying jobs-Architect (.36), Civil Engineer $(.40)$, and Securities Salesperson $(.26)$ - have the highest coordinates. Jobs that are low paying-Bookkeeper $(-.25)$ and Librarian $(-.44)$ - or low paying for the required amount of education-Counselor $(-.24)$ and Teacher $(-.26)$ - have the lowest coordinates. Because the ordering of stimuli along Dimension 4 roughly corresponds to their level of compen- sation, Dimension 4 can be interpreted as a monetary compensation dimension.

The interpretation of dimensions need not rely solely on subjective judgments. Correlational procedures can be used to confirm or disconfirm empirically the interpretation of a dimension (Davison, 1983). A full interpretation of the solution consists of identifying the features (such as the Holland categories) that correspond to the stimulus groupings in the solution space and identifying the attributes (such as pay or prestige) that correspond to the solution dimensions.

Participant weights. In a three-way or individual differences analysis, the output includes a table of participant weights, such as that in Table 3 . For each participant, this table will contain one weight for each dimension. Behind the weights lies the presumption that some dimensions will be more important than others for a given participant. For instance, Participant D in Table 3 has a larger weight (.50) along Dimension 4 than along any other dimension, suggesting that the Monetary Compensation Dimension figured most prominently in this participant's judgments. Furthermore, the presumption is that the most important dimension will not be the same for every participant. Whereas Participant D in Table 3 has the largest weight along Dimension $4(.50)$, Participant $\mathrm{A}$ has the largest weight along Dimension 1 (.49). In three-way or individual differences analyses, there is an attempt to quantify the importance of a dimension in the judgments of each participant as a way of describing individual differences between participants in the relative importance of dimensions. By describing differences in the importance of dimensions, the participant weights characterize differences in the way various participants seem to conceptualize the objects.

Fit measure. The fit measure indicates how well the stimulus coordinates account for the proximity data. The best-known fit measure is called STRESS, although not all MDS programs employ this statistic. STRESS equals 0.0 if the coordinates account for the data perfectly; it increases toward 1.0 as the data are less well accounted for. Some MDS analyses use correlation-like fit measures that equal 1.0 if the coordinates account for the data perfectly and decrease toward 0.0 as the data are less well accounted for.

In most applications, the fit measure is one consideration used in deciding how many dimensions are required to reproduce the structure of the objects. If the researchers do not know the appropriate number of dimensions, they can obtain several solutions, each with a different number of dimensions, and can choose between them on the basis of fit and other considerations. To compare their fit, one can form a fit by a dimensions plot, such as that in Figure 2. A clear elbow in the graph, if there is one, suggests that the appropriate number of dimensions is the number plotted on the horizontal axis below the elbow.

Fit is not the only consideration in deciding dimensionality. Before deciding how many dimensions to retain, users try to interpret the dimensions in the several solutions. One would favor the highest dimensional solution in which all dimensions

TABLE 3

Participant Weights for Five Participants

\begin{tabular}{cllll}
\hline \hline \multirow{2}{*}{ Subject } & \multicolumn{4}{c}{ Dimension } \\
\cline { 2 - 5 } & $\mathbf{1}$ & $\mathbf{2}$ & $\mathbf{3}$ & $\mathbf{4}$ \\
\hline A & .49 & .20 & .19 & .26 \\
B & .33 & .43 & .00 & .12 \\
C & .34 & .29 & .42 & .22 \\
D & .28 & .27 & .19 & .50 \\
E & .28 & .25 & .25 & .28 \\
\hline
\end{tabular}




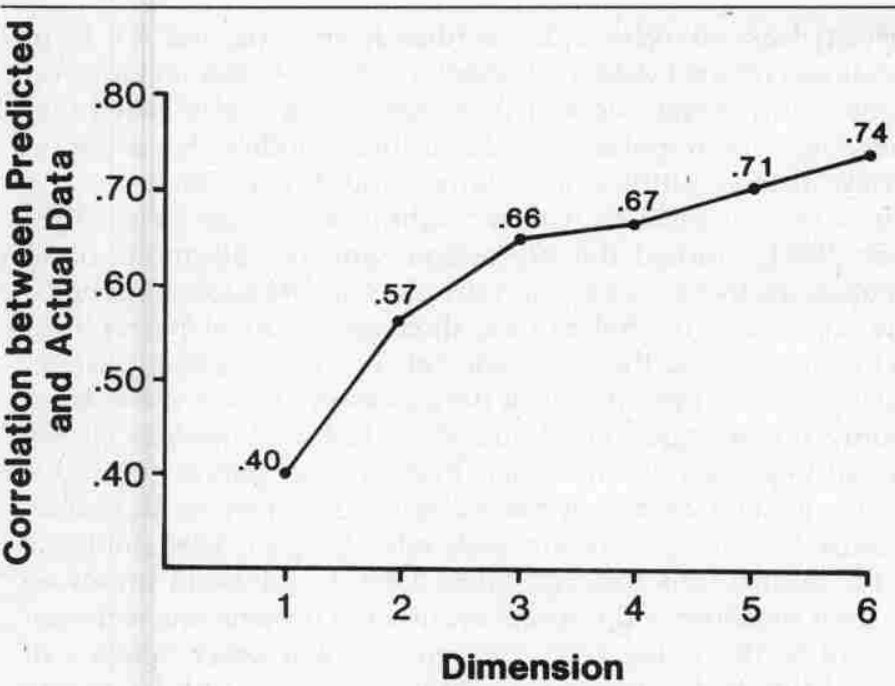

FIGURE 2

\section{Correlation Between Predicted and Actual Data Plotted Against Number of Dimensions}

can be interpreted. Considerations of dimension interpretability tend to override those of fit.

\section{Example}

This example was taken from Shubsachs and Davison's (1979) study of individual differences in perceptions of occupations. The authors assumed that one role of the vocational counselor is to help clients gain insight into the way they perceive occupations and the basis on which they differentiate among occupations. Because vocational counselors have studied occupations and their attributes, the authors expected that counselors would perceive occupations differently than would clients. Thus, they examined the occupational perceptions of vocational counselors and two potential client groups-undergraduate liberal arts students and engineering students.

The participants consisted of three samples: 32 male liberal arts students, 11 male engineering students, and 11 vocational experts, either vocational counselors or researchers. The experts were recruited from the Vocational Assessment Clinic of the Department of Psychology, University of Minnesota.

In the selection of occupations, each of Holland's six major categories were to be represented by an equal number of occupations. A sample size of 18 occupations allowed equal representation of categories without making the task of judging similarity unmanageable. Because most participants were men, the occupations were limited to those that men are likely to enter.

A task of judging direct similarity was used. Each participant rated each pair of occupations in terms of similarity on a scale much like the one shown above (the Librarian-Pharmacist rating). This task had two advantages for the study. First, it yielded a data matrix for each participant, as required by the three-way analysis the authors wished to employ. Second, the task of judging direct similarity left participants free to rate occupational similarity in terms of any attributes they desired. The task itself does not impose attributes.

As their method of analysis, the authors employed the threeway approach embodied in the INDSCAL computer program (Carroll \& Chang, 1970). One issue in the study concerned whether the three participant groups (liberal arts students, engineering students, and vocational experts) would differ in the importance they attached to various dimensions. The par- ticipant weight estimates provided by a three-way approach gave the authors a basis for comparing dimension importances across groups.

To decide how many dimensions to retain, Shubsachs and Davison began by obtaining solutions differing in dimensionality. The fit measures for these solutions are shown in Figure 2. If there is any elbow in this plot, it lies at the point labeled .66 directly above three dimensions along the horizontal axis. After trying to interpret the dimensions in the several solutions, the authors concluded that all four dimensions in the fourdimensional solution could be interpreted, and hence they retained a four-dimensional solution.

The stimulus coordinates from this study are provided in Table 2, and a portion of the configuration is plotted in Figure 1. The next step was to develop an interpretation for each dimension. In looking at the Dimension 1 coordinates in Table 2 and the horizontal axis in Figure 1, the authors concluded that this dimension primarily distinguished production-oriented jobs at the positive end-Civil Engineer (.31), Architect (.29), and Electrician (.25) - from sales-oriented jobs at the negative end-Securities Salesperson $(-.49)$, Auto Salesperson $(-.47)$, and Real Estate Agent $(-.40)$. Therefore, the authors labeled Dimension 1 the Production-Sales Dimension. Dimension 2 was characterized by scientific jobs at the positive end-Biostatistician (.36), Pharmacist (.31), and Computer Operator (.31) - and by artistic occupations at the negative end-Commercial Artist $(-.54)$, Writer $(-.33)$, and Architect $(-.25)$. Therefore, Dimension 2 was labeled the Scientific-Artistic Dimension.

Dimension 3 contained the two blue-collar occupations at the positive end-Aircraft Mechanic (.52) and Electrician (.32). The white-collar occupations were scattered over the remainder of the dimension. Therefore, Dimension 3 was labeled the BlueCollar-White-Collar Dimension. As discussed above, Dimension 4 was labeled the Compensation Dimension, with the high paying occupations at the positive end and the low paying occupations at the negative end.

Having interpreted all four dimensions, the authors turned to an inspection of the participant weight matrix, a portion of which is reproduced in Table 3 . Rather than interpreting the weights for individual participants, however, Shubsachs and Davison computed a mean participant weight for each group on the several dimensions. They found no significant differences in the mean weights along Dimensions 1-3, suggesting that the liberal arts students, engineering students, and vocational experts attached approximately equal importance to these dimensions in making their judgments. Along the fourth dimension, however, the means were $0.469,0.455$, and $0.365, F(2,51)=3.218$, $p<.05$, for the liberal arts, engineering, and expert groups, respectively, suggesting that the two student groups attached more importance to the Compensation Dimension than did the vocational experts.

Like some researchers before them and somewhat to their surprise, Shubsachs and Davison found few differences in occupational perceptions among people with seemingly quite different career paths. They attributed this finding to their use of a broad set of occupations. According to the authors, more marked individual differences may be observed by studying narrowly defined occupations in a specialized field as perceived by two groups-one group whose vocational background is similar to that of persons in the occupations studied and another group whose vocational background is dissimilar.

\section{Alternatives to Multidimensional Scaling}

In psychology, cluster analysis and factor analysis have been used as the main alternatives to MDS. Cluster analysis is used, as is MDS, to analyze proximity data so as to yield a representation of stimulus structure. Both methods have been applied 
to a variety of proximity measures (i.e., direct similarity judgments, probability-based measures, and profile similarity measures). Cluster analysis represents structure in terms of qualitative categories; MDS represents structure in terms of quantitative dimensions. If, for example, the stimuli were people, a cluster solution would be interpreted in terms of discrete, qualitative attributes such as marital status or religious affiliation, whereas some or all of the MDS dimensions would be interpreted in terms of quantitative variables such as age or intelligence. In cluster analysis the stimuli are divided into groups whose members are more like each other in some respects than they are like the members of the other groups. Because cluster analysis and MDS represent the structures differently, they are often viewed as complementary methods for highlighting different features of the stimuli. At times, both methods have been applied to the same data, with cluster analysis used to obtain a two-dimensional solution in which groupings could be displayed as in Figure 1.

Both factor analysis and MDS represent the structure of data in terms of quantitative dimensions, with factors in factor analysis corresponding to dimensions in MDS. Because it requires only ordinal data, nonmetric MDS has been viewed as appropriate for a wider variety of proximity data than is possible with factor analyses. Factor analysis has been generally limited to the analysis of correlation coefficients, whereas nonmetric MDS has been applied to the full range of proximity data described above. When nonmetric MDS and factor analysis have been applied to the same data (usually correlation coefficients), it often takes fewer dimensions than factors to represent the data. This makes graphic representation of MDS solutions simpler than those of factor analytic solutions (Davison, 1985; MacCallum, 1974; Schlessinger \& Guttman, 1969; Shepard, 1972). As yet, it is not clear whether the greater simplicity of MDS solutions is always a virtue. Proponents of MDS would argue that it fully represents the structure in simpler form. On the other hand, proponents of factor analysis might argue that MDS does not fully represent the structure.

\section{APPLICATIONS OF MULTIDIMENSIONAL SCALING}

In the past decade the use of MDS in counseling research has increased. A great many MDS applications are relevant for vocational counselors and researchers; MDS techniques can also be applied to family, group, and individual counseling issues. After reading this literature, we also concluded that MDS has potential applications in counseling practice.

\section{Vocational Counseling}

In vocational counseling, MDS has been used primarily to investigate the structure of vocational interests and the perceptions of occupations. For example, Rounds, Davison, and Dawis (1979) examined the fit of the six Strong-Campbell Interest Inventory (SCII) (Campbell, 1977) General Occupational Theme scales and the Vocational Preference Inventory (VPI) (Holland, 1965) scales to Holland's hexagonal model (Holland, 1973) of six personality types. Correlation matrices of the SCII and VPI occupational theme scales for both men and women were used as proximity data. It was found that for men, the SCII scale interrelationships conformed to Holland's RIASEC hexagonal model; for women, however, the SCII scales did not fit the hexagonal model as well. For the VPI, data for both the men and the women fit the model reasonably well.

This finding has implications for vocational counseling because the SCII manual instructs counselors to interpret the General Occupational Theme scales as measures of Holland's personality types; Rounds et al. (1979) found more support for this recommendation in their male sample than in their female sample and thus encouraged counselors to use caution in the practice of interpreting female scores according to Holland's typol- ogy. At least six other MDS studies have examined the fit of vocational interest data to Holland's or Roe's vocational interest models. In general, most of these studies have also called into question some important details of these models. For a critical review of these studies, see Rounds and Zevon (1983).

In a study similar to that of Shubsachs and Davison (1979), Reeb (1971) studied the perceptions and classifications of 15 occupations by 64 vocational counselors and 44 adolescent boys. The counselors tended to base their occupational perceptions on job prestige and the distinction between blue-collar and whitecollar jobs. The perceptions of the adolescent clients were based mainly on prestige and desirability. The middle-class clients tended to perceive white-collar, high-prestige jobs as desirable; the lower-class clients perceived blue-collar jobs as desirable. Because the clients and counselors rated the perceived similarity of the occupations through MDS, Reeb could avoid imposing his own preconceived notions about what dimensions were important to the rating task. He believed that other benefits of using MDS in his study were "the extreme simplicity of the dimensional structure" and the "intuitive understanding and economy and clarity of exposition as to how these groups saw these occupations" (Reeb, 1971, p. 242).

Multidimensional scaling has been used to investigate a number of other areas relevant to vocational counselors and researchers. For example, MDS studies have focused on the structure of job satisfaction, perceptions of vocational counseling roles, work outcomes, occupational reinforcers, potential work mobility, design of work environments, job analysis and classification, career preferences, and stages of vocational interest development.

\section{Family and Group Counseling}

Another use of MDS has been to assess family or group members' perceptions of each other. Sprouse and Brush (1980) were interested in the nature and development of group members' interpersonal perceptions. They examined (a) how the group members viewed one another, (b) the complexity of their perceptions, (c) the stability of their perceptions across the life of the group, and (d) the relative importance of the interpersonal dimensions at various points in the group members' lives. Eleven members of an 8-week therapy group for drunk driving offenders judged the similarity of each other at the end of each group session. These proximity data were analyzed using individual differences MDS.

The three major dimensions influencing group members' perceptions of each other were (a) high versus low self-disclosure about alcohol problems; (b) talkers versus nontalkers, and (c) race (Black versus White). The first dimension remained stable in its importance across the life of the group. The second dimension became more important and the third dimension less important. There was considerable individual variation in the salience of the dimensions. Blacks tended to place more weight on disclosure of the alcohol problem and on racial dimensions than did Whites. Both racial groups rated Dimension 2 (talkers versus nontalkers) about the same. As expected, the group leader placed more importance on Dimension 2.

This study provided information about the nature and development of the group members' interpersonal perceptions, about group structure, and about group process. In this study MDS was more useful as a technique than were most traditional sociometric techniques because it (a) enabled the researchers to discover the major underlying dimensions that influenced the group members' perceptions rather than selecting them a priori; (b) yielded a geometric representation of the groups' interpersonal structure, which allowed the investigators to interpret distances between group members meaningfully; (c) allowed the examination of individual differences in perception; and (d) 
showed whether the complexity or nature of the group members' perceptions changed over time. Also, MDS has been used to study (a) the nature and development of group members' perceptions of each other during the life of a T group, (b) the nature of family members' perceptions of each other before and during family therapy, and (c) the dimensions influencing group members' perceptions of each other in a self-analytic group.

\section{Individual Counseling}

At least a dozen MDS studies have implications for personal counseling and research. Horowitz (1979) examined the general categories of interpersonal problems presented by psychotherapy clients. Horowitz had 50 psychology students sort 127 "interpersonal problems" into categories of similarity. The interpersonal problems had been identified by expert raters who watched 28 videotaped, semistructured intake interviews of psychotherapy clients.

The proximity data were analyzed with nonmetric MDS, which revealed three major dimensions of clients' interpersonal problems. Dimension 1 was "degree of psychological involvement between the client and a significant other," Dimension 2 was "nature of the involvement (hostile versus friendly)," and Dimension 3 was "client's intention to influence, change, or control the significant other." Horowitz then used a hierarchical clustering scheme to classify the interpersonal problems. The combined results of the MDS and cluster analyses produced five thematic clusters of interpersonal problems (intimacy, aggression, compliance, independence, and sociability) located in different regions of the three-dimensional MDS space.

By revealing the underlying dimensions along which interpersonal problems are perceived to vary and by identifying specific clusters of problems, Horowitz provided conceptual clarity to an otherwise very confusing, unorganized domain. He also classified clients' presenting problems at a single level of abstraction-the interpersonal behavior level. Researchers and counselors could find their discussion of clients' presenting problems greatly facilitated should this lead, as Horowitz suggested, to a standardized interpersonal problem inventory. Whether or not this hope is realized, Horowitz's study is one example of how MDS can be used to investigate issues relevant to individual counselors. It has also been used to investigate such issues as power strategies in intimate relationships, implicit theories of psychopathology, individual differences in the perception and judgment of psychopathology, perceptions of complex assertive situations, implicit theories of personality, and perceived dimensions of interpersonal relations.

\section{Counseling Practice}

The research reviewed above illustrates the potential MDS has for contributing to counseling theory and research. It also suggests that MDS holds promise as a pre-interview assessment technique, as a tool for tracking outcomes of interventions, and as a client feedback mechanism. First, MDS's potential as a preinterview technique should be considered. To be fully effective, counselors need to understand clients' perceptions of the world. Counselors can use MDS to map clients' perceptions or cognitive representations of occupations, family members, interpersonal relationships, and other phenomena. These mappings or visual representations of clients' perceptions could help counselors better understand their clients. The MDS studies in person perception (reviewed by Jones, 1983) show the promise MDS has for mapping social structures, such as the family or therapy group.

Just as MDS can be used to chart the client's cognitive representations before counseling begins, it can be applied at various points during an intervention to trace changes in that representation. The Sprouse and Brush (1980) group therapy study represents the best illustration of MDS as a tool for tracing intervention outcomes.

Also, MDS can serve as a feedback mechanism for clients. Clients can gain insight from seeing a properly interpreted mapping of their cognitive representation. Viewing a map of a significant other's cognitive representation can help one understand the other, can present an alternative way to view the environment, or can open a dialogue with the other. Showing clients a map of how their perceptions are changing during an intervention may help them understand changes in themselves or in their environments.

Widespread clinical application of MDS requires translating methods developed in basic research into practical counseling tools. Microcomputer-based MDS systems represent one way of developing such tools. These systems should begin with data being collected from the client and should end with a narrative interpretation for the counselor.

In conclusion, the potential of MDS has yet to be fully realized in the counseling field. Nevertheless, the MDS research to date suggests that MDS, coupled with the ingenuity of counseling researchers and practitioners, will make a valuable contribution to counseling research, theory, and practice.

\section{REFERENCES}

Campbell, D.P. (1977). Manual for the Strong-Campbell Interest Inventory (2nd ed.). Stanford, CA: Stanford University Press.

Carroll, J.D., \& Chang, J.J. (1970). Analysis of individual differences in multidimensional scaling via $N$-way generalization of "Eckart-Young" decomposition. Psychometrika, 35, 283-319.

Coxon, A.P.M. (1982). The user's guide to multidimensional scaling. Exeter, NH: Heinemann Educational Books.

Davison, M.L. (1983). Multidimensional scaling. New York: Wiley.

Davison, M.L. (1985). Multidimensional scaling vs. components analysis of test intercorrelations. Psychological Bulletin, 97, 94-105.

Davison, M.L., Robbins, S., \& Swanson, D. (1978). Stage structure in objective moral judgements. Developmental Psychology, 14, 137-146.

Gazda, G.M., \& Mobley, J.A. (1981). INDSCAL multidimensional scaling. Journal of Group Psychotherapy, Psychodrama, and Sociometry, 34, $54-73$.

Holland, J.L. (1965). Manual for the Vocational Preference Inventory (6th ed.). Palo Alto, CA: Consulting Psychologists Press.

Holland, J.L. (1973). Making vocational choices: A theory of careers. Englewood Cliffs, NJ: Prentice-Hall.

Horowitz, L.M. (1979). On the cognitive structure of interpersonal problems treated in psychotherapy. Journal of Consulting and Clinical Psychology, 47, 5-15.

Jepsen, D.A., \& Grove, W.M. (1981). Stage order and dominance in adolescent vocational decision-making processes: An empirical test of the Tiedeman-O'Hara paradigm. Journal of Vocational Behavior, 18, 237251.

Jones, L.E. (1983). Multidimensional models of social perception, cognition, and behavior. Applied Psychological Measurement, 7, 451-472.

Kruskal, J.B., \& Wish, M. (1978). Multidimensional scaling. Beverly Hills, CA: Sage.

Kruskal, J.B., Young, F.W., \& Seery, J.B. (1973). How to use KYST, a very flexible program to do multidimensional scaling and unfolding. Unpublished manuscript, Bell Laboratories, Murray Hills, NI

Lingoes, J.C. (1973). The Guttman-Lingoes nonmetric program series. Ann Arbor, MI: Mathesis Press.

MacCallum, R.C. (1974). Relations between factor analysis and multidimensional scaling. Psychological Bulletin, 81, 505-516.

Pruzansky, S. (1975). How to use SINDSCAL: A computer program for individual differences in multidimensional scaling. Unpublished manuscript, Bell Laboratories, Murray Hills, NJ.

Ramsey, J.O. (1978). MULTISCALE: Four programs for multidimensional scaling by the method of maximum likelihood. Chicago: International Educational Services.

Reeb, M. (1971). Multidimensional perceptions by counselors and 14year-old boys of suitability for jobs, and their prestige and desirability. Occupational Psychology, 45, 233-242.

Rosenberg, S. (1982). The method of sorting in multivariate research with applications selected from cognitive psychology and person perception. In N. Hirschberg \& L. G. Humphreys (Eds.), Multivariate applications in the social sciences. Hillsdale, NJ: Erlbaum.

Rounds, J.B., Jr., Davision, M.L., \& Dawis, R.V. (1979). The fit between Strong-Campbell Interest Inventory general occupational themes and Holland's hexagonal model. Journal of Vocational Behavior, 15, 303-315. 
Rounds, J.B., Jr. \& Zevon, M.A. (1983). Multidimensional scaling research in vocational psychology. Applied Psychological Measurement, 7, 491-510.

Schiffman, S.S., Reynolds, M.L., \& Young, F.W. (1981). Introduction to multidimensional scaling. New York: Academic Press.

Schlessinger, I.M., \& Guttman, L. (1969). Smallest space analysis of intelligence and achievement tests. Psychological Bulletin, 71, 95-100.

Shepard, R.N. (1972). Introduction to Volume I. In R.N. Shepard, A.K. Romney, \& S.B. Nerlove (Eds.), Multidimensional scaling: Theory and applications in the behavioral sciences (Vol. 1, pp. 1-20). New York: Seminar Press.

Shoben, E.J. (1983). Applications of multidimensional scaling in cognitive psychology. Applied Psychological Measurement, 7, 473-490.

Shubsachs, A.P.W., \& Davison, M.L. (1979). Individual differences in perceptions of occupations and occupational reinforcers. Journal of Occupational Psychology, 52, 299-310.
Sprouse, C.L., \& Brush, D.H. (1980). Assessment of interpersonal perception: Assessing a quasi-therapy group by individual differences multidimensional scaling. Small Group Behavior, 11, 35-49.

Young, F.W., \& Lewyckyj, R. (1979). ALSCAL 4 user's guide (2nd ed.). Chapel Hill, NC: Data Analysis and Theory Associates.

Mark L. Davison is a professor in the Department of Educational Psychology, University of Minnesota, Minneapolis. P. Scott Richards is a predoctoral counseling intern in the University Counseling Services, University of Minnesota. James B. Rounds, Jr., is an assistant professor in the Department of Counseling and Educational Psychology, State University of New York at Buffalo. Acknowledgment is due to the Spencer Foundation and the National Academy of Education for their support of James B. Rounds during the period in which this article was written. 